\title{
Uric acid and hypertension
}

\author{
Miguel A. Lanaspa ${ }^{1}$ Ana Andres-Hernando ${ }^{1} \cdot$ Masanari Kuwabara ${ }^{1,2}$ \\ Key words Uric acid $\cdot$ Hypertension $\cdot$ Risk factor $\cdot$ Causality $\cdot$ Mechanism
}

Received: 22 November 2019 / Revised: 28 November 2019 / Accepted: 28 November 2019 / Published online: 8 June 2020

(c) The Japanese Society of Hypertension 2020

The association between serum uric acid levels and high blood pressure in humans is well established. For example, a cross-sectional study determined that each $1 \mathrm{mg} / \mathrm{dL}$ increase in serum uric acid contributes a $20 \%$ increased prevalence of hypertension in a general population not treated with hyperuricemia and hypertension [1]. Similarly, in longitudinal cohort studies, asymptomatic hyperuricemia without comorbidities predicts the development of hypertension [2]. Moreover, hyperuricemia also contributes to the development of hypertension from prehypertension [3]. Based on these observations, the lowering of serum uric acid levels has become an intriguing therapeutic approach in hypertension. In this regard, several randomized placebo control trials (RCTs) have shown that the reduction of uric acid, either with a xanthine oxidase inhibitor (allopurinol) or with a uricosuric agent (probenecid), substantially decreased blood pressure in adolescents $[4,5]$. Similarly, a recent RCT also showed that pegloticase, a recombinant uricase conjugated to polyethylene glycol, significantly decreased blood pressure compared with placebo in subjects with chronic refractory gout [6]. From these studies, a direct causal relationship between serum uric acid and blood pressure could be extrapolated. However, not all RCTs conducted have been able to elucidate such a clear causal role for uric acid in hypertension. For example, in the FEATHER study, febuxostat did not significantly decrease blood pressure compared with placebo in subjects with asymptomatic hyperuricemia and stage 3 chronic kidney diseases [7]. Moreover, most Mendelian randomized studies

Masanari Kuwabara

kuwamasa728@gmail.com

1 Division of Renal Diseases and Hypertension, School of Medicine, University of Colorado Denver, Denver, CO, USA

2 Intensive Care Unit and Department of Cardiology, Toranomon Hospital, Tokyo, Japan showed a negative relationship between hyperuricemia and hypertension [8]. However, it is important to note that Mendelian studies involve gene-dependent associations, and even though hyperuricemia has an important genetic component, it is primarily caused by life habits and diet. Nevertheless, the current evidence suggests the need for further studies about the relationship between serum uric acid and hypertension, and specifically studies focusing on life habits, including drinking alcohol, such as the Saku study recently published in this journal.

The Saku study was conducted retrospectively using the cohort data (a mean of 4.0 years follow-up) to evaluate the risk factors for high uric acid with hypertension [9]. The study group enrolled 7848 participants who had undergone a medical checkup. After adjustment for alcohol consumption, hyperuricemia was found to be independently associated with the development of hypertension, with hazard ratios (HRs) of 1.37 in men and 1.54 in women. Furthermore, among nonalcohol drinkers, hyperuricemia was also independently associated with the development of hypertension, with HRs of 1.29 in men and 1.57 in women. Importantly, the interaction between hyperuricemia or alcohol consumption and sex was not significant. Based on these observations, the authors concluded that hyperuricemia could predict the development of hypertension independently of alcohol intake. Thus, the novelty of this study relates to the finding that serum uric acid is still associated with the development of hypertension even after excluding the effects of alcohol consumption on serum uric acid [10], which is a well-known risk factor for hyperuricemia. In addition, the strength of the study relies on its analysis with well-adjusted cofactors, including age, estimated glomerular filtration rate, diabetes, dyslipidemia, body mass index, smoking status, physical activity, family history, and systolic blood pressure. However, the limitation of the study is the lack of detailed medication data. It is known that some antihypertensive agents, such as thiazide, increase serum uric acid levels, while other medications, 
Fig. 1 The mechanisms by which hyperuricemia causes hypertension; crystal (extracellular uric acid) pathway and crystal-independent (intracellular uric acid) pathway.

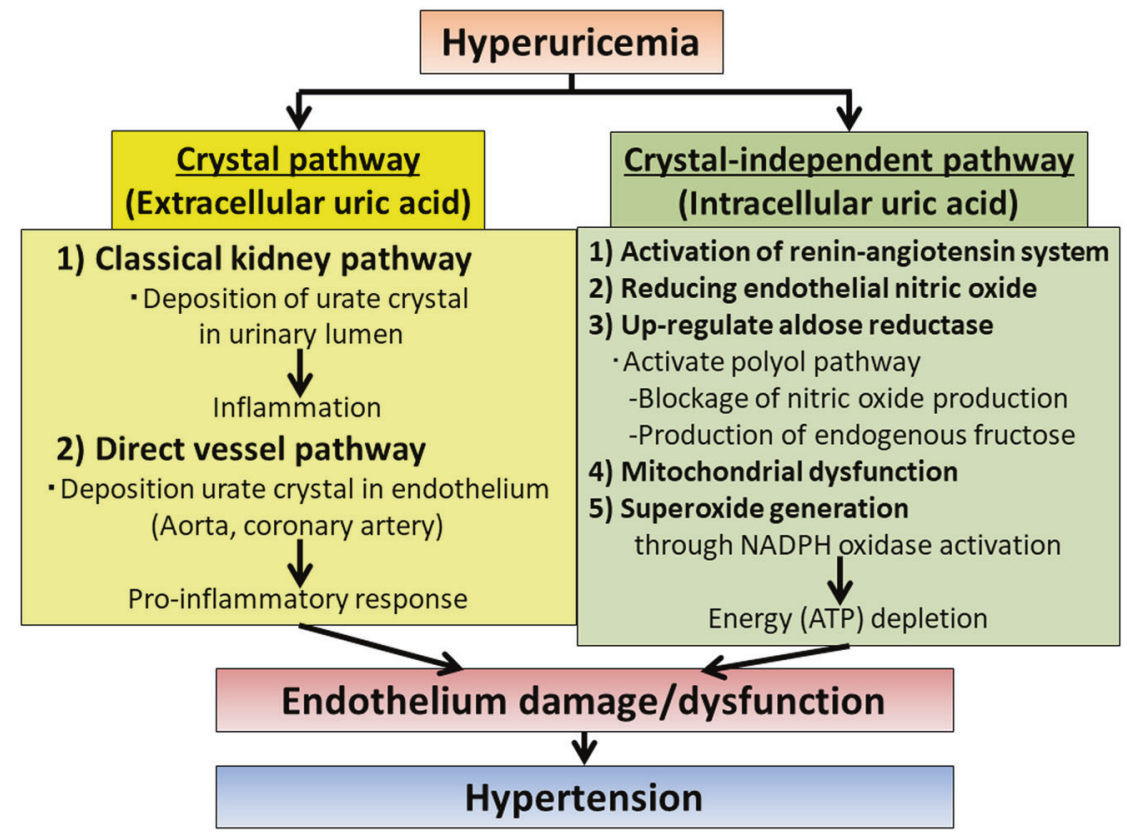

including losartan, fenofibrate, and sodium-glucose cotransporter-2 (SGLT2) inhibitors, decrease serum uric acid levels. The use of these medications in the population analyzed should have been taken into consideration. Moreover, observational studies such as the Saku study, although clinically relevant, are not designed to address whether there is causality between serum uric acid and hypertension. Therefore, there is still the need to identify the potential molecular and cellular mechanisms whereby hyperuricemia causes hypertension in basic and clinical studies.

Classically, the proposed mechanism whereby uric acid can cause hypertension relates to its primary deleterious effects on the kidney. These mechanisms include the activation of the intrarenal renin-angiotensin system and the deposition of urate crystals in the urinary lumen. However, recent evidence indicates that uric acid can cause direct endothelial injury and dysfunction. In this regard, Klauser et al. demonstrated the presence of urate deposits in the aorta and coronary arteries of patients with gout by dual-energy computer tomography and their association with higher coronary calcium score [11]. The deposition of urate crystals in main vessels may trigger a similar proinflammatory response as it is observed in the kidney, thus causing more direct endothelial damage [12]. A crystal-independent mechanism has also been postulated. It is well known that uric acid impairs endothelial function by reducing endothelial nitric oxide synthase phosphorylation under hypoxic conditions [13]. Moreover, recently, several groups have found that soluble uric acid upregulates the expression of aldose reductase in the endothelium and other tissues [14, 15]. Interestingly, the elevation of aldose reductase expression results in the activation of the polyol pathway, leading to two main consequences: the blockade of nitric oxide production and the production of endogenous fructose. Both of these mechanisms seem to play an important deleterious role in the pathogenesis of high blood pressure in the endothelium as the blockade of aldose reductase or fructokinase, the enzyme involved in the metabolism of fructose, markedly improves endothelial cell function $[15,16]$. The molecular mechanism whereby uric acid upregulates aldose reductase and causes endothelial cell dysfunction seems to be mediated by its prooxidant properties. More specifically, it has been shown that uric acid induces mitochondrial dysfunction and superoxide generation through the activation of nicotinamide adenine dinucleotide phosphate (NADPH) oxidases, thus depleting energy (adenosine triphosphate (ATP)) capacity [17]. Of interest, mitochondrial ATP production is important for proper endothelial signaling and function. In summary, these studies highlight new evidence of the direct deleterious effects of uric acid on the endothelium, which may be important underlying factors in the pathogenesis of hypertension (Fig. 1) [18, 19].

\section{Compliance with ethical standards}

Conflict of interest MK reports receiving a research grant from Toranomon Hospital, Okinaka Memorial Institute for Medical Research, and the Gout Research Foundation in Japan. The remaining authors have nothing to disclose.

Publisher's note Springer Nature remains neutral with regard to jurisdictional claims in published maps and institutional affiliations. 


\section{References}

1. Kuwabara M, Niwa K, Nishi Y, Mizuno A, Asano T, Masuda K, et al. Relationship between serum uric acid levels and hypertension among Japanese individuals not treated for hyperuricemia and hypertension. Hypertens Res. 2014;37:785-9.

2. Kuwabara M, Niwa K, Hisatome I, Nakagawa T, Roncal-Jimenez CA, Andres-Hernando A, et al. Asymptomatic hyperuricemia without comorbidities predicts cardiometabolic diseases: five-year Japanese cohort study. Hypertension. 2017;69:1036-44.

3. Kuwabara M, Hisatome I, Niwa K, Hara S, Roncal-Jimenez CA, Bjornstad $\mathrm{P}$, et al. Uric acid is a strong risk marker for developing hypertension from prehypertension: a 5-year Japanese cohort study. Hypertension. 2018;71:78-86.

4. Soletsky B, Feig DI. Uric acid reduction rectifies prehypertension in obese adolescents. Hypertension. 2012;60:1148-56.

5. Feig DI, Soletsky B, Johnson RJ. Effect of allopurinol on blood pressure of adolescents with newly diagnosed essential hypertension: a randomized trial. JAMA. 2008;300:924-32.

6. Johnson RJ, Choi HK, Yeo AE, Lipsky PE. Pegloticase treatment significantly decreases blood pressure in patients with chronic gout. Hypertension. 2019;74:95-101.

7. Kimura K, Hosoya T, Uchida S, Inaba M, Makino H, Maruyama $\mathrm{S}$, et al. Febuxostat therapy for patients with Stage 3 CKD and asymptomatic hyperuricemia: a randomized trial. Am J Kidney Dis. 2018;72:798-810.

8. Sedaghat S, Pazoki R, Uitterlinden AG, Hofman A, Stricker BH, Ikram MA, et al. Association of uric acid genetic risk score with blood pressure: the Rotterdam study. Hypertension. 2014;64:1061-6

9. Tatsumi Y, Asayama K, Morimoto A, Satoh M, Sonoda N, Miyamatsu N, et al. Hyperuricemia predicts the risk for developing hypertension independent of alcohol drinking status in men and women: the Saku study. Hypertens Res. 2020; 43:442-49.

10. Kuwabara M. Hyperuricemia, cardiovascular disease, and hypertension. Pulse. 2016;3:242-52.
11. Klauser AS, Halpern EJ, Strobl S, Gruber J, Feuchtner G, Bellmann-Weiler R, et al. Dual-energy computed tomography detection of cardiovascular monosodium urate deposits in patients with gout. JAMA Cardiol. 2019. https://doi.org/10.1001/jamaca rdio.2019.3201.

12. Kuwabara M, Kanbay M, Hisatome I. Uric acid and hypertension because of arterial stiffness. Hypertension. 2018;72:582-4.

13. Otani N, Toyoda S, Sakuma M, Hayashi K, Ouchi M, Fujita T, et al. Effects of uric acid on vascular endothelial function from bedside to bench. Hypertens Res. 2018;41:923-31.

14. Sanchez-Lozada LG, Andres-Hernando A, Garcia-Arroyo FE, Cicerchi C, Li N, Kuwabara M, et al. Uric acid activates aldose reductase and the polyol pathway for endogenous fructose and fat production causing development of fatty liver in rats. J Biol Chem. 2019;294:4272-81.

15. Huang Z, Hong Q, Zhang X, Xiao W, Wang L, Cui S, et al. Aldose reductase mediates endothelial cell dysfunction induced by high uric acid concentrations. Cell Commun Signal. 2017;15:3.

16. Lanaspa MA, Kuwabara M, Andres-Hernando A, Li N, Cicerchi C, Jensen T, et al. High salt intake causes leptin resistance and obesity in mice by stimulating endogenous fructose production and metabolism. Proc Natl Acad Sci USA. 2018;115:3138-43.

17. Lanaspa MA, Sanchez-Lozada LG, Choi YJ, Cicerchi C, Kanbay $\mathrm{M}$, Roncal-Jimenez CA, et al. Uric acid induces hepatic steatosis by generation of mitochondrial oxidative stress: potential role in fructose-dependent and -independent fatty liver. J Biol Chem. 2012;287:40732-44.

18. Sanchez-Lozada LG, Lanaspa MA, Cristobal-Garcia M, GarciaArroyo F, Soto V, Cruz-Robles D, et al. Uric acid-induced endothelial dysfunction is associated with mitochondrial alterations and decreased intracellular ATP concentrations. Nephron Exp Nephrol. 2012;121:e71-78.

19. Wilson C, Lee MD, Heathcote HR, Zhang X, Buckley C, Girkin $\mathrm{JM}$, et al. Mitochondrial ATP production provides long-range control of endothelial inositol trisphosphate-evoked calcium signaling. J Biol Chem. 2019;294:737-758. 